Article

\title{
Global Analysis for an HIV Infection Model with CTL Immune Response and Infected Cells in Eclipse Phase
}

\author{
Karam Allali $^{1} \mathbb{B}^{\mathbb{D}}$, Jaouad Danane ${ }^{1} \mathbb{C D}^{\mathbb{D}}$ and Yang Kuang ${ }^{2, *}$ \\ 1 Laboratory of Mathematics and Applications, Faculty of Sciences and Technologies, \\ University Hassan II of Casablanca, P.O. Box 146, Mohammedia 20650, Morocco; \\ allali@fstm.ac.ma or allali@hotmail.com (K.A.); jaouaddanane@gmail.com (J.D.) \\ 2 School of Mathematical and Statistical Sciences, Arizona State University, Tempe, AZ 85287, USA \\ * Correspondence: kuang@asu.edu
}

Received: 27 July 2017; Accepted: 17 August 2017; Published: 21 August 2017

\begin{abstract}
A modified mathematical model describing the human immunodeficiency virus (HIV) pathogenesis with cytotoxic T-lymphocytes (CTL) and infected cells in eclipse phase is presented and studied in this paper. The model under consideration also includes a saturated rate describing viral infection. First, the positivity and boundedness of solutions for nonnegative initial data are proved. Next, the global stability of the disease free steady state and the endemic steady states are established depending on the basic reproduction number $R_{0}$ and the CTL immune response reproduction number $R_{C T L}$. Moreover, numerical simulations are performed in order to show the numerical stability for each steady state and to support our theoretical findings. Our model based findings suggest that system immunity represented by CTL may control viral replication and reduce the infection.
\end{abstract}

Keywords: CTL immune response; global stability; HIV infection model; viral dynamics

\section{Introduction}

Human immunodeficiency virus (HIV) is known as a pathogen causing the acquired immunodeficiency syndrome (AIDS), which is the end-stage of the infection. After that, the immune system fails to play its life-sustaining role [1,2]. Indeed, it is well known that the cellular immune responses play an indispensable role during the HIV viral infection, especially in people known as elite controllers who are infected by HIV but maintain a normal CD4 count for many years and remain asymptomatic or have a very delayed disease progression over the course of their HIV infection $[3,4]$.

The basic viral infection model with CTLs immune was first studied in [5] and its global stability was studied in [6,7]. A prevailing theory suggests that CTL cells are responsible for the sudden decrease HIV virus load and the subsequent quasi-steady state level of the viral load after a sharp increase during the primary infection phase [8-11]. In [12], the authors consider the interaction between CTL, viruses and macrophages and study the global stability in a model with a mass action infection term. With a saturated infection term, the same mathematical question was tackled in [13] with a model consisting of four ordinary differential equations with a Beddington-DeAngelis infection term.

More recently, the model describing the interaction between the HIV viruses, CD4 ${ }^{+} \mathrm{T}$ cells, infected cells with a standard incidence rate function describing saturated viral infection is formulated and studied in [14]. The authors study the local and global stability of the endemic states and illustrate that their model can fit well with some clinical HIV infection data sets. In this paper, we extend their work by incorporating the cytotoxic $\mathrm{T}$ lymphocytes (CTL) immune response. The dynamics 
of HIV infection with CTL response that we consider is given by the following nonlinear system of differential equations:

$$
\left\{\begin{array}{l}
\dot{x}=\lambda-d_{1} x-\frac{k_{1} x v}{x+v}, \\
\dot{s}=\frac{k_{1} x v}{x+v}-d_{2} s-k_{2} s, \\
\dot{y}=k_{2} s-d_{3} y-p y z, \\
\dot{v}=a y-d_{4} v, \\
\dot{z}=c y z-b z,
\end{array}\right.
$$

with the initial conditions $x(0)=x_{0}, s(0)=s_{0}, y(0)=y_{0}, v(0)=v_{0}$ and $z(0)=z_{0}$.

In this model, $x, y, s, v$ and $z$ denote the concentration of uninfected cells, infected cells, exposed cells, free virus and CTL cells, respectively. Susceptible host cells $\mathrm{CD} 4^{+} \mathrm{T}$ cells are produced at a rate $\lambda$, die at a rate $d_{1} x$ and become infected by virus at a rate $\frac{k_{1} x v}{x+v}$. Exposed cells die at a rate $d_{2} s$ and become infected at a rate of $k_{2} s$. Infected cells increase at rate $k_{2} s$ and die at rate $d_{3} y$ and are killed by the CTL response at a rate pyz. Free virus is produced by infected cells at a rate ay and decays at a rate $d_{4} v$. Finally, CTLs expand in response to viral antigen derived from infected cells at a rate $c y z$ and decay in the absence of antigenic stimulation at a rate $b z$. Note that this model (1) employs the more realistic standard incidence rate function $\frac{k_{1} x v}{x+v}$ as in $[14,15]$, which better describes the rate of viral infection. The schematic representing the viral dynamics of the problem under consideration is illustrated in Figure 1.

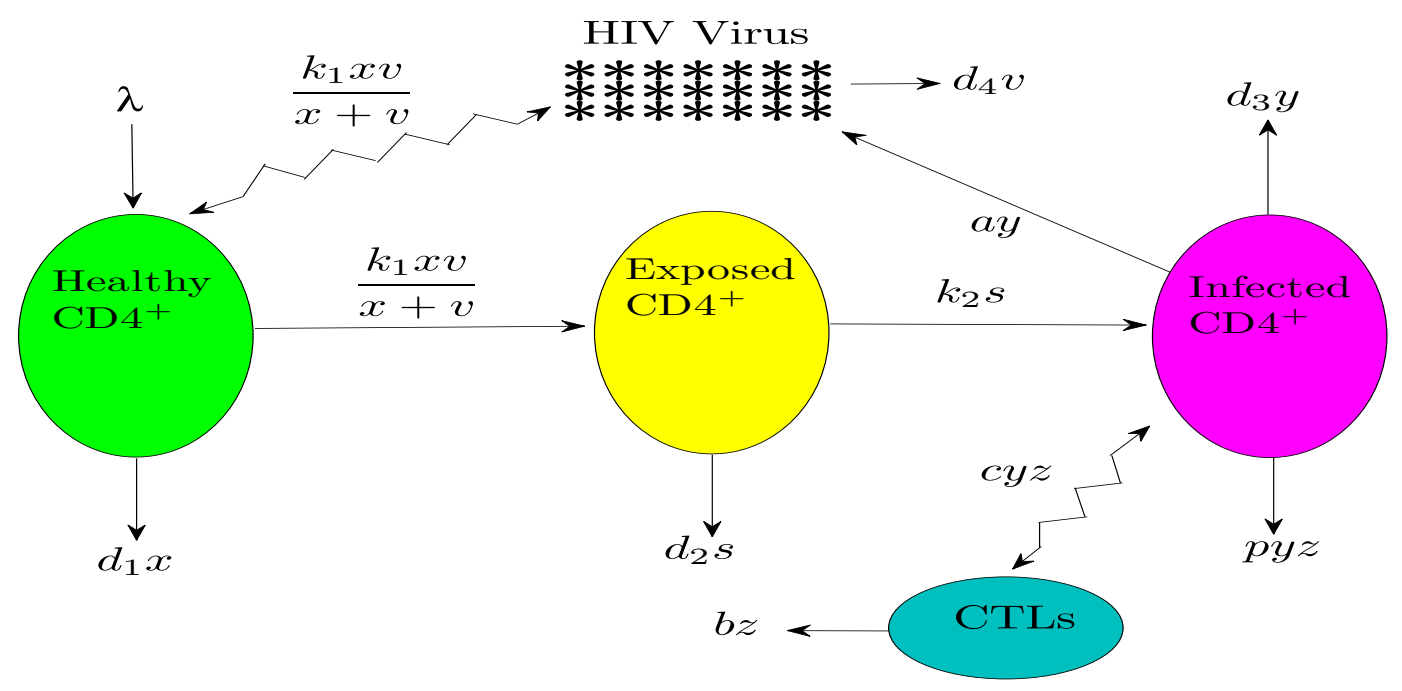

Figure 1. Schematic of the model under consideration.

The rest of the paper is organized as follows. In Section 2, we study the positivity and boundedness of solutions. We present the steady states and give a local stability result in Section 3. Section 4 is dedicated to the global stability results. Numerical simulations are performed in Section 5 and we conclude the paper in the last section.

\section{Mathematical Analysis of the HIV Model}

\subsection{Existence and Local Stability}

For the problems deal with cell population evolution, the cell densities should remain non-negative and bounded. In this section, we will establish the positivity and boundedness of solutions of the 
model (1). First of all, for biological reasons, the parameters $x_{0}, s_{0}, y_{0}, v_{0}$ and $z_{0}$ must be larger than or equal to 0 . Hence, we have the following result:

Proposition 1. For any non-negative initial conditions $\left(x_{0}, s_{0}, y_{0}, v_{0}, z_{0}\right)$, system (1) has a unique solution. Moreover, this solution is non-negative and bounded for all $t \geq 0$.

Proof. The proof is given in Appendix A.

Moreover, system (1) has an infection-free equilibrium $E_{f}=\left(\frac{\lambda}{d_{1}}, 0,0,0,0\right)$, corresponding to the maximal level of healthy $\mathrm{CD}^{+}$T-cells. The basic reproduction number of our problem is given as follows:

$$
R_{0}=k_{1} \frac{k_{2}}{d_{2}+k_{2}} \frac{a}{d_{3}} \frac{1}{d_{4}}
$$

where $\frac{k_{2}}{d_{2}+k_{2}}$ is the proportion of exposed cells to become productively infected cells, $\frac{a}{d_{3}}$ is the number of free virus production by an infected cell and $\frac{1}{d_{4}}$ is the average life of virus. From a biological point of view, $R_{0}$ stands for the average number of secondary infections generated by one infected cell when all cells are susceptibles. Depending on the value of this basic reproduction number $R_{0}$; in other words, depending on these three biological proportions, we will study the stability of the free-endemic and the endemic equilibria.

In addition to the disease free equilibrium, system (1) has three endemic equilibria. The first of them is $E_{1}=\left(x_{1}, s_{1}, y_{1}, v_{1}, z_{1}\right)$, where

$$
\begin{aligned}
& x_{1}=\frac{\lambda}{d_{1}+k_{1}\left(1-\frac{1}{R_{0}}\right)}, \\
& s_{1}=\frac{k_{1} \lambda R_{0}\left(1-\frac{1}{R_{0}}\right)}{\left(d_{2}+k_{2}\right)\left(d_{1}+k_{1}\left(1-\frac{1}{R_{0}}\right)\right)\left(\left(1-\frac{1}{R_{0}}\right) R_{0}+1\right)}, \\
& y_{1}=\frac{d_{4} \lambda R_{0}\left(1-\frac{1}{R_{0}}\right)}{a d_{1}+a k_{1}\left(1-\frac{1}{R_{0}}\right)} \\
& v_{1}=\frac{\lambda R_{0}\left(1-\frac{1}{R_{0}}\right)}{d_{1}+k_{1}\left(1-\frac{1}{R_{0}}\right)} \\
& z_{1}=0 .
\end{aligned}
$$

The second endemic steady state is $E_{2}=\left(x_{2}, s_{2}, y_{2}, v_{2}, z_{2}\right)$, where

$$
\begin{aligned}
& x_{2}=\frac{-a b d_{1}-a b k_{1}+\lambda c d_{4}+\sqrt{A}}{2 c d_{1} d_{4}}, \\
& s_{2}=\frac{d_{3} R_{0}}{k_{2}} \frac{b\left(-a b d_{1}-a b k_{1}+\lambda c d_{4}+\sqrt{A}\right)}{c\left(a b d_{1}-a b k_{1}+\lambda c d_{4}+\sqrt{A}\right)}, \\
& y_{2}=\frac{b}{c^{\prime}} \\
& v_{2}=\frac{b a}{c d_{4}}, \\
& z_{2}=\frac{d_{3}\left(\left(R_{0}-1\right)\left(-a k_{1} b+\lambda c d_{4}+\sqrt{A}\right)-a b d_{1}\left(R_{0}+1\right)\right)}{p\left(a b d_{1}-a k_{1} b+\lambda c d_{4}+\sqrt{A}\right)} .
\end{aligned}
$$


The third endemic steady state is $E_{3}=\left(x_{3}, y_{3}, v_{3}, z_{3}\right)$, where

$$
\begin{aligned}
& x_{3}=-\frac{a b d_{1}+a b k_{1}-\lambda c d_{4}+\sqrt{A}}{2 c d_{1} d_{4}}, \\
& s_{3}=\frac{k_{1} x_{3} v_{3}}{\left(k_{2}+d_{2}\right)\left(x_{3}+v_{3}\right)}, \\
& y_{3}=\frac{b}{c} \\
& v_{3}=\frac{b a}{c d_{4}} \\
& z_{3}=\frac{k_{2} s_{3}-d_{3} y_{3}}{p y_{3}}
\end{aligned}
$$

with $A=\left(a b k_{1}-\lambda c d_{4}\right)^{2}+a^{2} b^{2} d_{1}^{2}+2 a^{2} b^{2} d_{1} k_{1}+2 \lambda a b c d_{1} d_{4}$.

The last endemic steady-state $E_{3}$ will not be taken under consideration since $x_{3}<0$, which is not possible biologically. On the other hand, from the components of $E_{1}$, it is clear that, when $R_{0}>1$, this endemic point exists. In what follows, we will make use of the following CTL immune response reproduction number $R_{C T L}$ to classify the model system dynamics

$$
R_{C T L}=\frac{c y_{1}}{b}=\frac{c d_{4} \lambda R_{0}\left(1-\frac{1}{R_{0}}\right)}{a b d_{1}+a b k_{1}\left(1-\frac{1}{R_{0}}\right)},
$$

depending on the value of this CTL immune reproduction number $R_{C T L}$; in other words, depending on $R_{0}$ and the parameters describing the effectiveness of CTL like $c$ and $b$, we will study the stability of the endemic equilibria.

First, observe that the second endemic state $E_{2}=\left(x_{2}, y_{2}, v_{2}, z_{2}\right)$ exists when $R_{C T L}>1$. This endemic state is also called an interior equilibrium.

We explain the existence of this endemic equilibrium $E_{2}$ as follows. We recall first that, in this state, both of the free viruses and CTL cells are present. Assume that $R_{0}>1$, in the total absence of CTL immune response, the infected cell load per unit time is $\frac{d_{4} \lambda R_{0}\left(1-\frac{1}{R_{0}}\right)}{a d_{1}+a k_{1}\left(1-\frac{1}{R_{0}}\right)}$. Via the five equations of model (1), CTL cells reproduced due to infected cells stimulating per unit time is $\frac{c d_{4} \lambda R_{0}\left(1-\frac{1}{R_{0}}\right)}{a d_{1}+a k_{1}\left(1-\frac{1}{R_{0}}\right)}=c y_{1}$. The CTL load during the lifespan of a CTL cell is $\frac{c d_{4} \lambda R_{0}\left(1-\frac{1}{R_{0}}\right)}{a b d_{1}+a b k_{1}\left(1-\frac{1}{R_{0}}\right)}=R_{C T L}$. If $\frac{c d_{4} \lambda R_{0}\left(1-\frac{1}{R_{0}}\right)}{a b d_{1}+a b k_{1}\left(1-\frac{1}{R_{0}}\right)}>1$, we will have the existence of the endemic equilibrium $E_{2}$.

The local stability result of the disease-free equilibrium is given as follows.

Proposition 2. The disease-free equilibrium, $E_{f}$, is locally asymptotically stable for $R_{0}<1$.

Proof. The proof is given in Appendix A.

The local stability of the other steady states is quite similar to the first one. Hence, the rest of the work will be devoted only to the global stability analysis of the problem under consideration.

\subsection{Global Stability Results}

This subsection deals with the global stability analysis of the disease-free and the endemic equilibria. Our approach involves the construction of appropriate Lyapunov functions. First, we have the following global stability result of the disease-free equilibrium. 
Proposition 3. If $R_{0} \leq 1$, then the endemic point $E_{f}$ is globally stable.

Proof. The proof is given in Appendix B.

For the global stability result of the endemic equilibrium $E_{1}$, we have the following.

Proposition 4. If $R_{0} \geq 1$ and $R_{C T L} \leq 1$, then the endemic point $E_{1}$ is globally stable.

Proof. The proof is given in Appendix B.

Finally, the global stability result concerning the last endemic point $E_{2}$ is given as follows.

Proposition 5. If $R_{0} \geq 1$ and $R_{C T L} \geq 1$, then the endemic point $E_{2}$ is globally stable.

Proof. The proof is given in Appendix B.

\section{Numerical Results and Simulations}

In order to perform the numerical simulations, system (1.1) will be solved numerically using the fourth order Runge-Kutta iterative scheme. The parameter values or ranges used are presented in Table 1.

Table 1. Parameters, their symbols and default values used in the suggested HIV model.

\begin{tabular}{cccc}
\hline Parameters & Meaning & Value & References \\
\hline$\lambda$ & Source rate of CD4 $4^{+}$T cells & {$[0,10]$} & {$[16]$} \\
$k_{1}$ & Average of infection & {$\left[2.5 \times 10^{-4}, 0.5\right]$} & {$[14]$} \\
$d_{1}$ & Decay rate of healthy cells & 0.0139 & {$[14]$} \\
$d_{2}$ & Death rate of exposed CD4 ${ }^{+} \mathrm{T}$ cells & 0.0495 & {$[14]$} \\
$k_{2}$ & The rate that exposed become infected CD4 ${ }^{+}$T cells & 1.1 & {$[14]$} \\
$d_{3}$ & Death rate of infected CD4 $4^{+}$T cells, not by CTL killing & 0.5776 & {$[14]$} \\
$a$ & The rate of production the virus by infected CD4 $4^{+}$T cells & {$[2,1250]$} & {$[14]$} \\
$d_{4}$ & Clearance rate of virus & {$[0.3466,2.4]$} & {$[14]$} \\
$p$ & Clearance rate of infection & 0.0024 & {$[17]$} \\
$c$ & Activation rate CTL cells & 0.15 & {$[17]$} \\
$b$ & Death rate of CTL cells & 0.5 & {$[17]$} \\
\hline
\end{tabular}

Figure 2 shows the behavior of infection during the first 60 days. For the parameters used in this figure, the basic reproduction number is $R_{0}=0.2209<1$; we clearly see that the solutions of the system converge to the disease-free equilibrium point $E_{f}=(827.22,0,0,0,0)$. This numerical result is consistent with the theoretical result concerning the stability of $E_{f}$. In addition, the plots showing the stability of this disease-free point in phase plane are given in Figure 3.

Figure 4 shows the behavior of the infection for the first sixty days. The chosen parameters in this figure ensure that the basic reproduction number is greater than unity $\left(R_{0}=11.049>1\right)$ and the immune response reproduction number is less than unity $\left(R_{C T L}=3.596 \times 10^{-1}<1\right)$. It is clearly seen that, in this case, all the solutions converge towards the CTL-free endemic equilibrium $E_{1}=\left(19.96,5.98 \times 10^{-1}, 1.14,199.78,0\right)$ that agrees with our theoretical finding concerning the stability of $E_{1}$. Moreover, the plots showing the stability of this CTL-free endemic point in phase plane are given in Figure 5.

Finally, Figure 6 illustrates the disease dynamics when both the basic reproduction number and the immune response reproduction number are greater than unity. Indeed, for the chosen parameters in this figure, we have $R_{0}=11.049>1$ and $R_{C T L}=4.13>1$. It can be seen that the infection persists and the convergence towards the infection steady state $E_{3}=(285.12,6.55,3.33,555.55,660.86)$ is observed. This numerical result is in good agreement with the theoretical result concerning the stability of the 
last endemic equilibrium. The plots showing the stability of this final endemic point in phase plane are given in Figure 7. In these two last figures, we clearly observe the importance of CTL immune response in maximizing the healthy cells and reducing the viral load. It is also noteworthy that the CTLs do not vanish in this last case, which means that CTL cells will be always present when the infection becomes chronic.
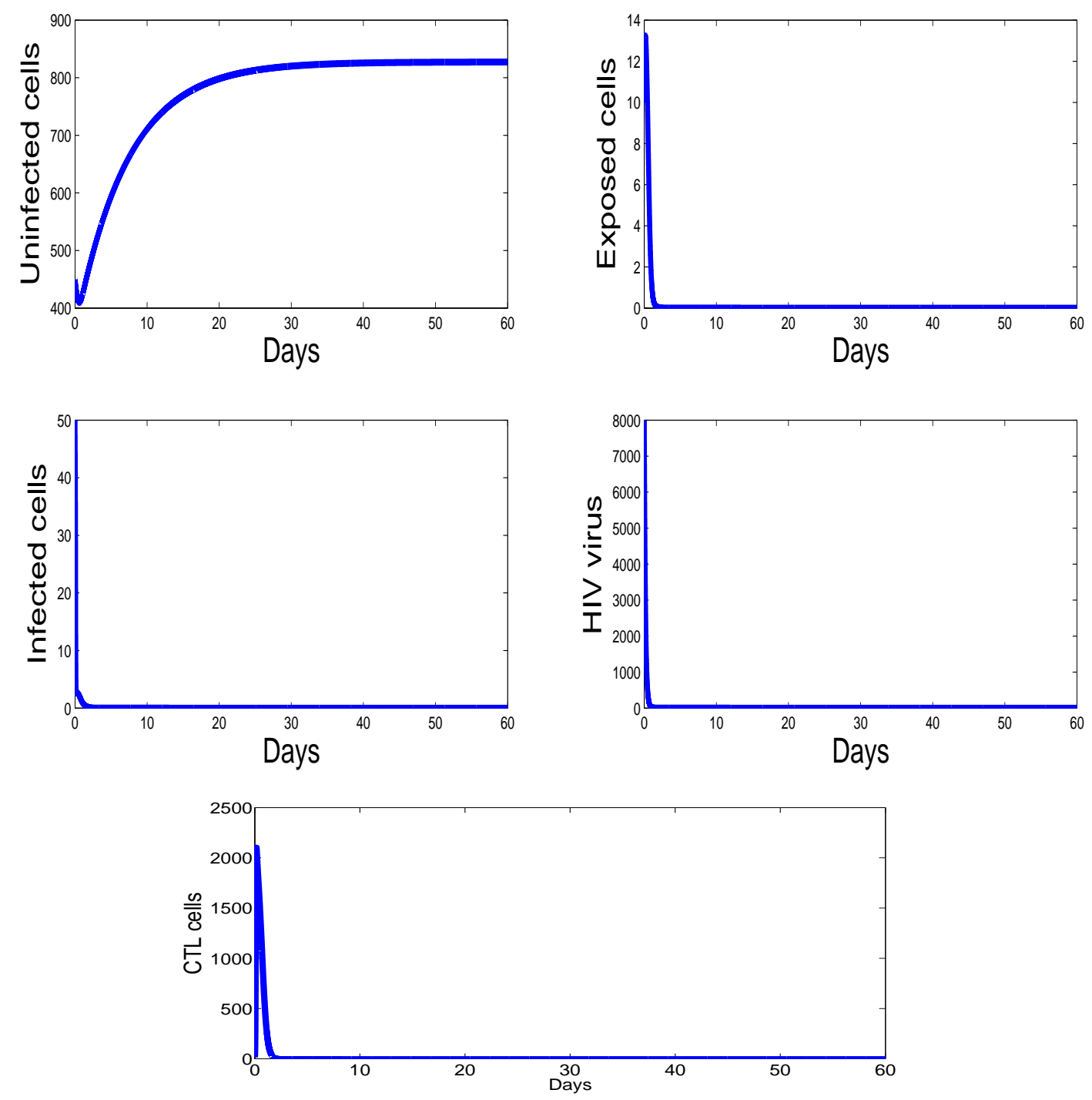

Figure 2. The behavior of the infection dynamics for $\lambda=10, d_{1}=0.0139, k_{1}=0.04, d_{2}=0.0495$, $k_{2}=1.1, d_{3}=0.5776, a=2, d_{4}=0.6, p=0.0024, c=0.15, b=0.5$. For the parameters used in this figure, the basic reproduction number is $R_{0}=0.2209<1$. Solutions of the system converge to the disease-free equilibrium point $E_{f}=(827.22,0,0,0,0)$. 

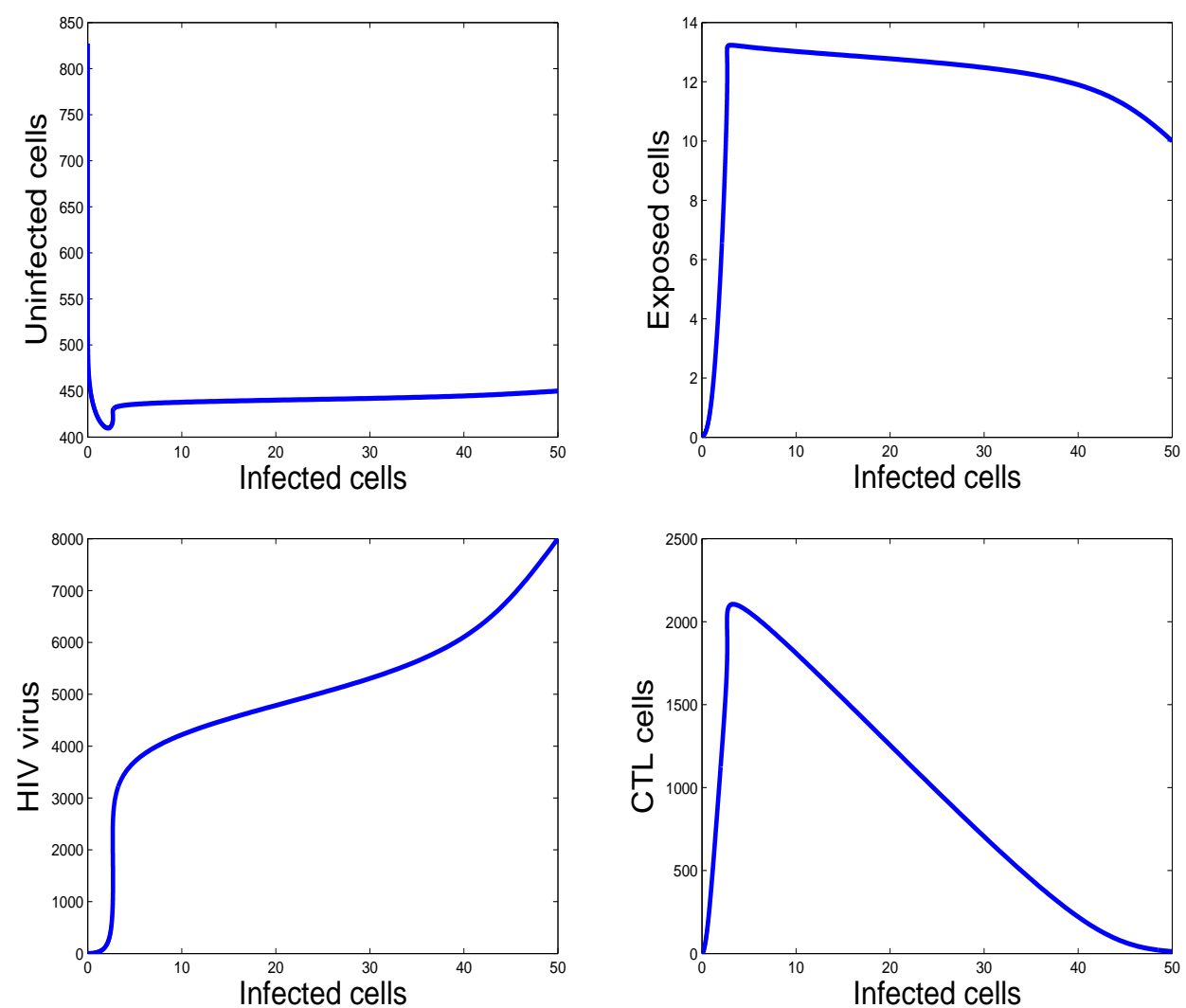

Figure 3. Selective phase portraits to illustrate the solution behavior of the infection dynamics when $\lambda=10$, $d_{1}=0.0139, k_{1}=0.04, d_{2}=0.0495, k_{2}=1.1, d_{3}=0.5776, a=2, d_{4}=0.6, p=0.0024, c=0.15, b=0.5$.
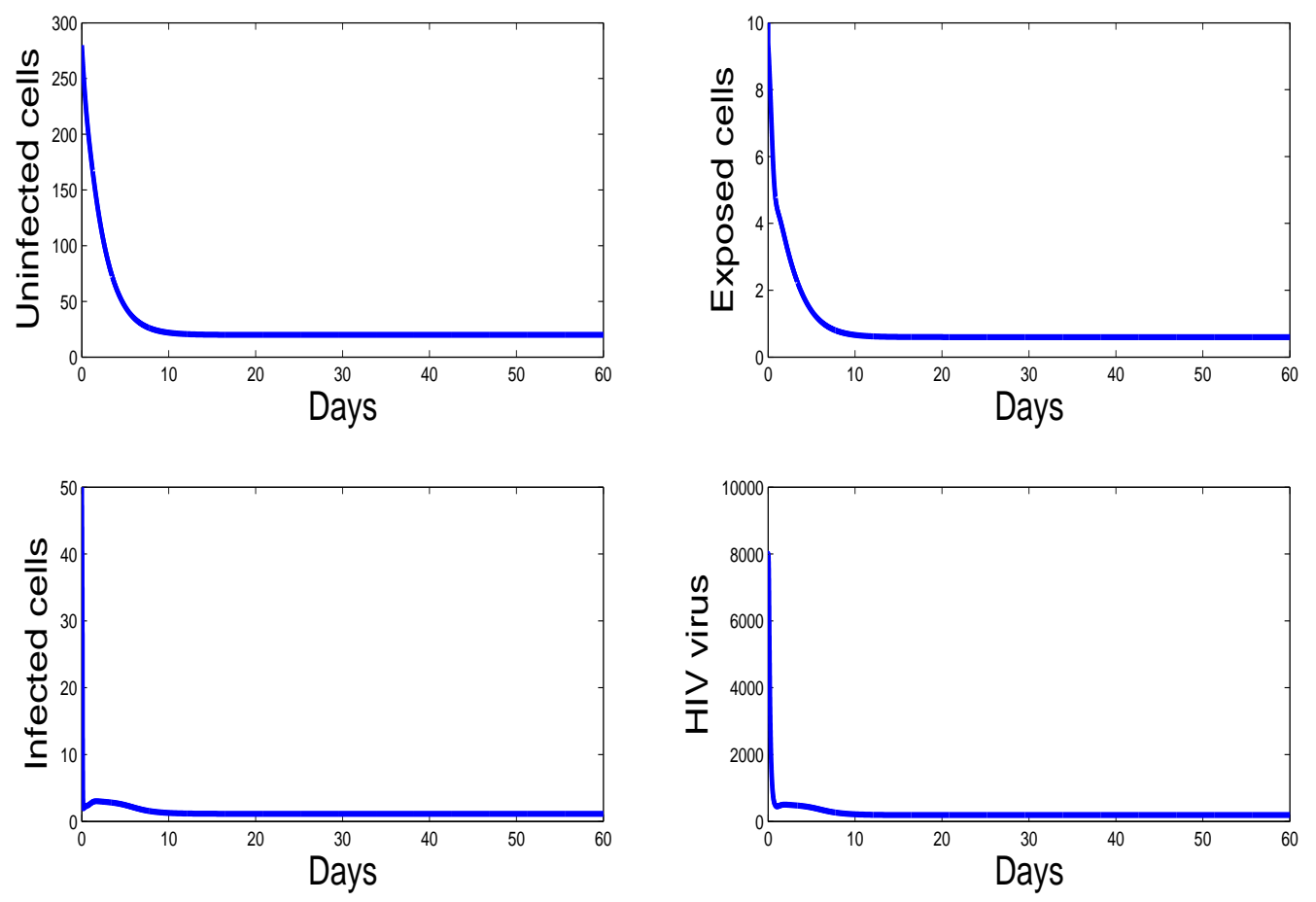

Figure 4. Cont. 


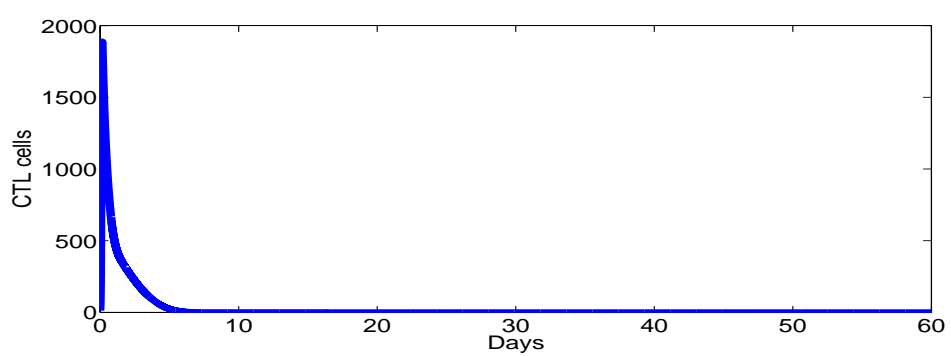

Figure 4. The behavior of the infection dynamics for $\lambda=1, d_{1}=0.0139, k_{1}=0.04, d_{2}=0.0495$, $k_{2}=1.1, d_{3}=0.5776, a=100, d_{4}=0.6, p=0.0024, c=0.15, b=0.5$. For the parameters used in this figure, the basic reproduction number is $11.049>1$. The immune response reproduction number $R_{C T L}=3.596 \times 10^{-1}<1$. In this case, all the solutions converge towards the endemic equilibrium $E_{1}=\left(19.96,5.98 \times 10^{-1}, 1.14,199.78,0\right)$.
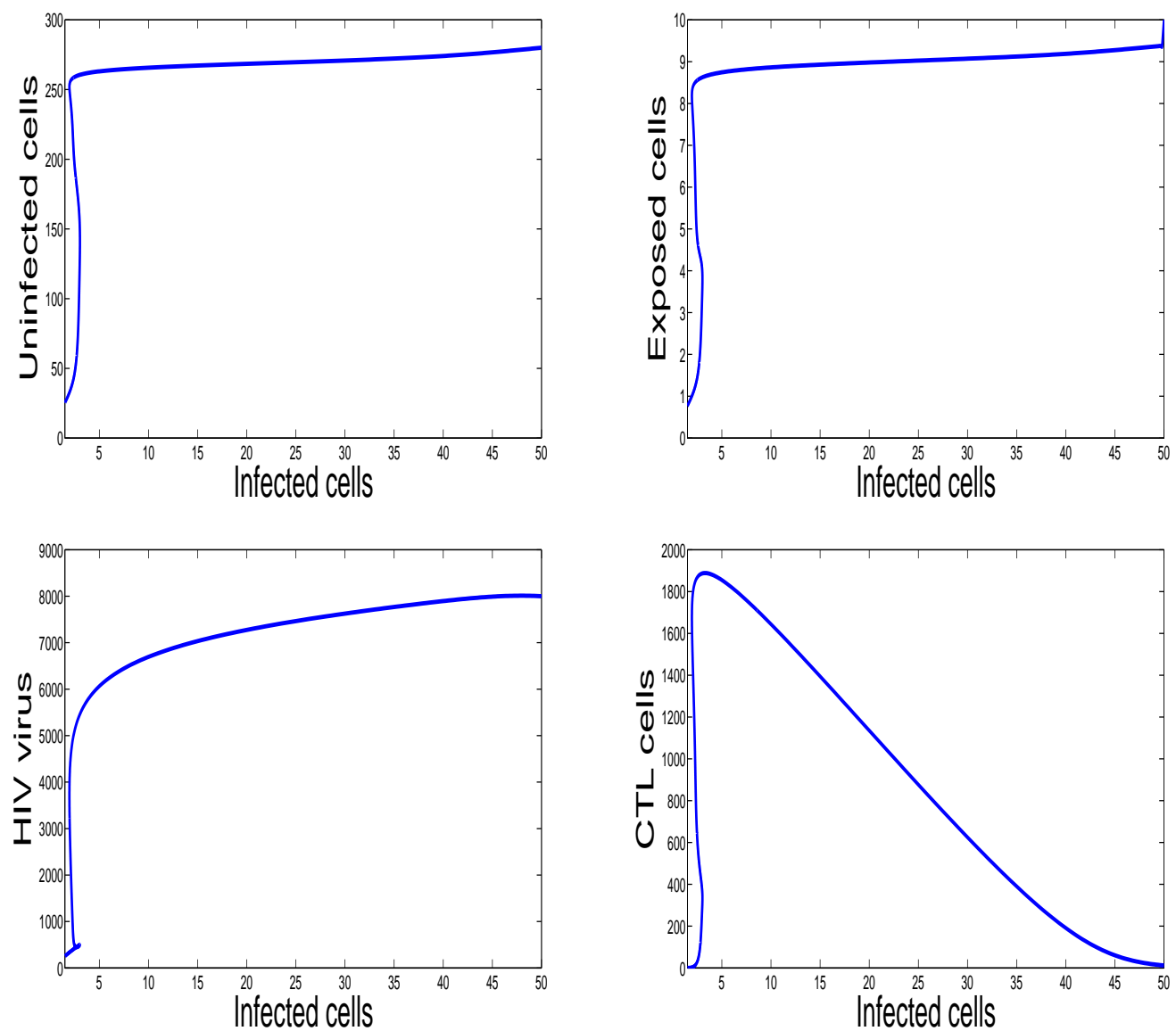

Figure 5. Selective phase portraits to illustrate the solution behavior of the infection dynamics when $\lambda=1, d_{1}=0.0139, k_{1}=0.04, d_{2}=0.0495, k_{2}=1.1, d_{3}=0.5776, a=100, d_{4}=0.6, p=0.0024$, $c=0.15, b=0.5$. 

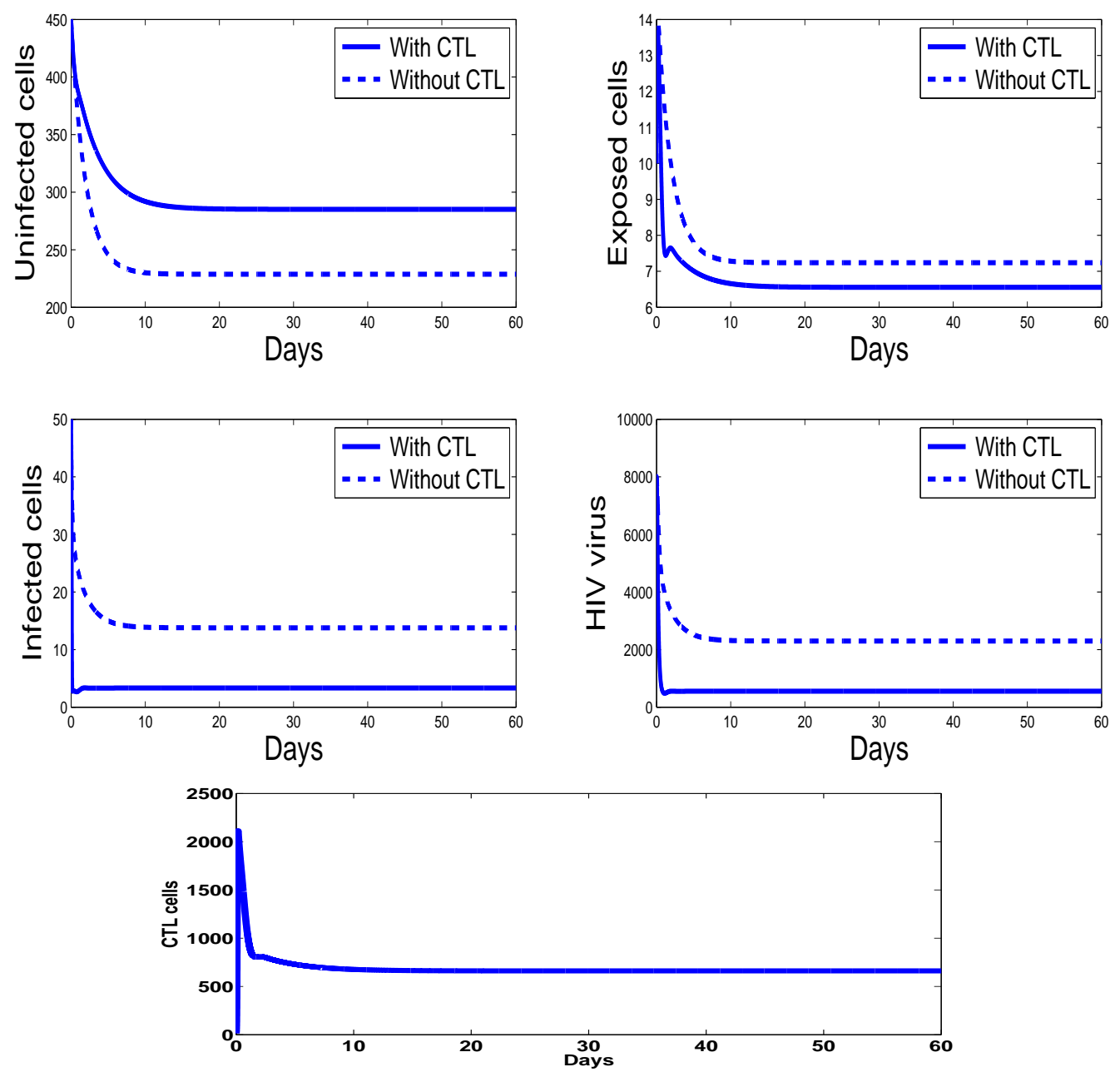

Figure 6. The behavior of the infection dynamics for $\lambda=10, d_{1}=0.0139, k_{1}=0.04, d_{2}=0.0495$, $k_{2}=1.1, d_{3}=0.5776, a=100, d_{4}=0.6, p=0.0024, c=0.15, b=0.5$. For the chosen parameters in this figure, we have $R_{0}=11.049>1$ and $R_{C T L}=4.13>1$. Solutions tending to the infection steady state $E_{3}=(285.12,6.55,3.33,555.55,660.86)$ are observed.
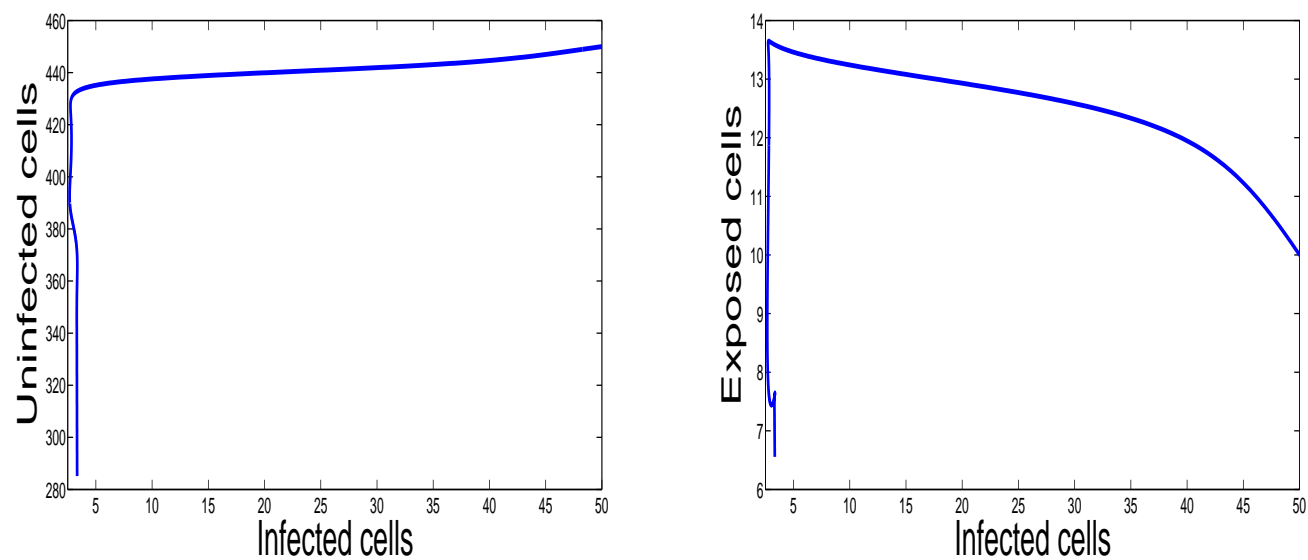

Figure 7. Cont. 

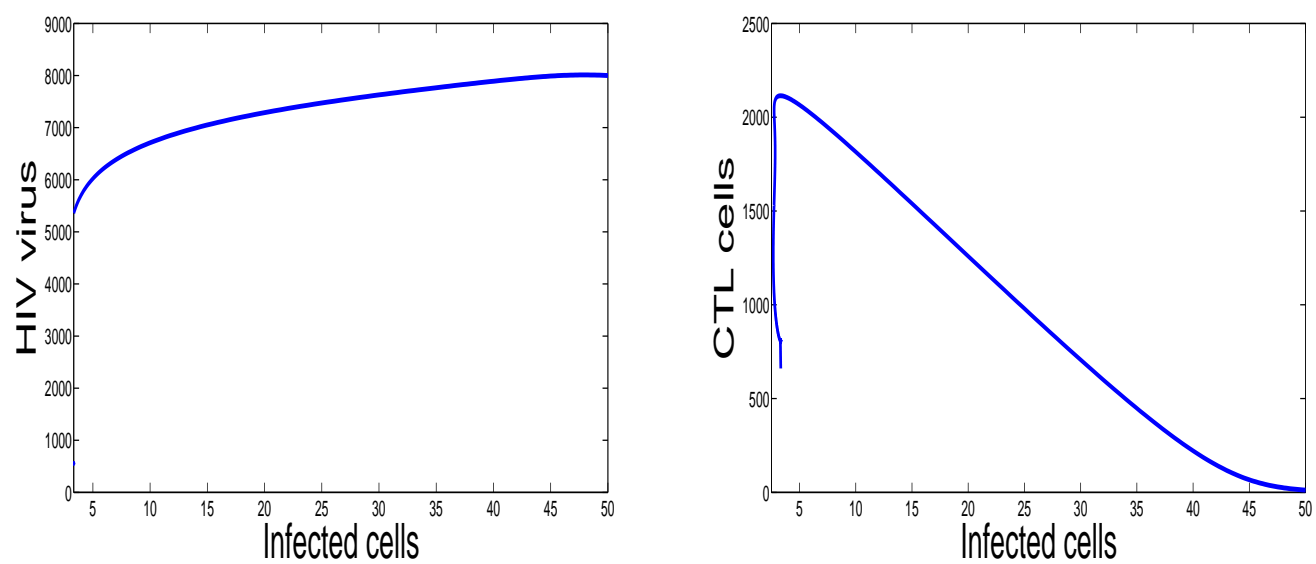

Figure 7. Selective phase portraits to illustrate the solution behavior of the infection dynamics when $\lambda=10, d_{1}=0.0139, k_{1}=0.04, d_{2}=0.0495, k_{2}=1.1, d_{3}=0.5776, a=100, d_{4}=0.6, p=0.0024$, $c=0.15, b=0.5$.

\section{Discussion}

The HIV dynamics involving the density uninfected cells, the density of the infected cells, the density of HIV virus and the amount of CTL cells have been widely studied by means of a different mathematical model starting from the work in 1996 by Nowak and Bangham [5] and two years later in 1998 by [18]. Later, many mathematical approaches were developed in order to better understand the complex dynamics of the fatal HIV disease.

Recently, in 2015, Conway and Perelson [19] studied the same issue by incorporating the treatment into the HIV model. Motivated by all those previous works treating the dynamics of HIV along with the CTL immune response, we have incorporated the CTL effect to a model suggested recently in 2015 by Sun et al. [14], in which the authors studied HIV dynamics by incorporating into the model the treatment and a more realistic incidence functional that describes the infection rate taking into account the crowd of the free viruses near the uninfected cells. In the latter work, the uninfected cells need time to become infected after HIV virus contact. Indeed, there exists an eclipse phase that is needed for the exposed CD4 ${ }^{+} \mathrm{T}$ cells to become infected (see Figure 1). In this paper, we have taken into account this phase and we have added the CTL immune response to the model.

In this paper, we have established the well-posedness of the suggested model that ensures that all the solutions exist and are bounded. This agrees with the biological reality that the total cell amounts of these populations are bounded. Moreover, three steady states of the problem are found and their local and global stability results are determined depending on the basic reproduction number $R_{0}$ and the CTL immune response reproduction number $R_{C T L}$.

We have also presented some numerical results that confirm our theoretical findings. In addition, several plots of the solutions in phase diagrams have been drawn in order to illustrate the stability of the three steady states.

The model dynamics indicate that the immune response represented by the cytotoxic $\mathrm{T}$ lymphocytes is efficient in controlling viral replication. Indeed, our numerical results show that, in the endemic case (see Figure 6), the CTL immune response plays an essential role in maximizing the effect of the uninfected $\mathrm{CD} 4^{+} \mathrm{T}$ cells, minimizing the amount of the infected cells and reducing the HIV viral load.

\section{Conclusions}

In this paper, we have studied the model of the HIV dynamics in the presence of the immune response, which is represented by the cytotoxic T lymphocytes (CTL) cells. The model describes the interaction between the healthy cells, the infected cells in eclipse phase, the productively infected 
cells and the immune cells. The model includes also a saturated rates in order to better describe the viral infection. The positiveness and the boundedness of solutions are established. In addition, we have studied the stability of both disease-free equilibrium and endemic equilibria. The disease free steady state is locally and globally asymptotically stable when the basic reproduction number is less than unity $\left(R_{0}<1\right)$. The existence of two other infection steady states when $R_{0}>1$ is established. The global stability of these endemic steady states depends on the basic reproduction number $R_{0}$ and the CTL immune response reproduction number $R_{C T L}$. Numerical simulations are performed in order to show the behavior of infection during the days of observation. The theoretical and the numerical results are in good agreement. In addition, our model based results suggest that the system immunity represented by CTL can control viral replication and reduce the infection under appropriate conditions.

Acknowledgments: The authors would like to thank the three reviewers for their many helpful suggestions. Karam Allali is grateful to the support from the Moroccan American Commission for Educational and Cultural Exchange through the Fulbright Program and the hospitality of the School of Mathematical and Statistical Sciences of Arizona State University. Yang Kuang is partially supported by US NSF grants DMS-1518529 and DMS-1615879.

Author Contributions: Karam Allali and Yang Kuang discussed the idea of the work during the visit of the first at the Arizona State University. Karam Allali, Jaouad Danane and Yang Kuang wrote the paper. Karam Allali and Jaouad Danane carried out the numerical simuations.

Conflicts of Interest: The authors declare no conflict of interest.

\section{Appendix A. Existence and Local Stability Results Proof}

The proof of the existence result (Proposition 1) is as follows. First, by the classical differential equations theory, we can confirm that system (1) has a unique local solution $(x(t), s(t), y(t), v(t), z(t))$ in $\left[0, t_{m}\right)$.

First, the solution $x(t)$ is strictly positive for all $t \in\left[0, t_{m}\right)$. Indeed, assume the contrary, and let $t_{1}>0$ be the first time such that $x\left(t_{1}\right)=0$ and $\dot{x}\left(t_{1}\right) \leq 0$. From the first equation of system (1), we have $x\left(t_{1}\right)=\lambda>0$, which presents a contradiction. Therefore, $x(t)>0$ for all $t \in\left[0, t_{m}\right)$. We also have the following:

$$
\begin{gathered}
\left.\dot{s}\right|_{s=0}=\frac{k_{1} x v}{x+v} \geq 0, \\
\left.\dot{y}\right|_{y=0}=k_{2} s \geq 0, \\
\left.\dot{v}\right|_{v=0}=a y \geq 0,
\end{gathered}
$$

and

$$
\left.\dot{z}\right|_{z=0}=0 \geq 0 .
$$

This shows that $s(t) \geq 0, y(t) \geq 0, v(t) \geq 0$ and $z(t) \geq 0$ for all $t \in\left[0, t_{m}\right)$. On the other hand, for the boundedness of the solutions, assume first that

$$
X=x+s+y
$$

then, we will have

$$
\begin{aligned}
\dot{X} & =\lambda-d_{1} x-d_{2} s-d_{3} y-p y z, \\
& \leq \lambda-\delta X
\end{aligned}
$$

where $\delta=\min \left(d_{1}, d_{2}, d_{3}\right)$. Therefore,

$$
\begin{aligned}
X(t) & \leq X(0) e^{-\delta t}+\frac{\lambda}{\delta}\left(1-e^{-\delta t}\right) \\
& \leq X(0) e^{-\delta t}+\frac{\lambda}{\delta}
\end{aligned}
$$


By the same reasoning, concerning the fourth equation, we will have

$$
v(t) \leq v(0)+\frac{a}{d_{4}}\|y\|_{\infty} .
$$

For the last equation, we will have

$$
\begin{aligned}
\dot{z}(t)+b z(t) & =c y(t) z(t) \\
& =\frac{c}{p}\left(\lambda-\left(\dot{x}(t)+d_{1} x\right)-\left(\dot{s}(t)+\left(d_{2}+k_{2}\right) s\right)-\left(\dot{y}(t)+d_{3} y\right)\right),
\end{aligned}
$$

from which, we will have

$$
\begin{aligned}
z(t) & =\left(z(0)+\frac{c}{p}\left(x(0)+s(0)+y(0)-\frac{\lambda}{b}\right)\right) e^{-b t} \\
& +\frac{c}{p}\left(\frac{\lambda}{b}+\int_{0}^{t}\left(\left(b-d_{1}\right) x+\left(b-\left(d_{2}+k_{2}\right) s\right)+\left(b-d_{3}\right) y\right) e^{b(\xi-t)} d \xi-x-s-y\right) \\
& \leq\left(z(0)+\frac{c}{p}(x(0)+s(0)+y(0))\right)+\frac{c}{p}\left(\frac{\lambda}{b}+\frac{1}{b} \max \left(0 ; b-d_{1}\right)\right)\|x\|_{\infty} \\
& \left.+\max \left(0 ; b-\left(d_{2}+k_{2}\right)\right)\|s\|_{\infty}+\max \left(0 ; b-d_{3}\right)\|y\|_{\infty}\right)
\end{aligned}
$$

this proves that the solutions $x(t), s(t), y(t), v(t)$ and $z(t)$ are bounded. Hence, every local solution can be prolonged up to any time $t_{m}>0$, which means that the solution exists globally.

For the local stability result (Proposition 2), the proof is stated as follows.

At the disease-free equilibrium, $E_{f}$, the Jacobian matrix is given as follows:

$$
J_{E_{f}}=\left(\begin{array}{clrrc}
-d_{1} & 0 & 0 & -k_{1} & 0 \\
0 & -k_{2}-d_{2} & 0 & k_{1} & 0 \\
0 & k_{2} & -d_{3} & 0 & 0 \\
0 & 0 & a & -d_{4} & 0 \\
0 & 0 & 0 & 0 & -b
\end{array}\right) .
$$

The characteristic polynomial of $J_{E_{f}}$ is

$$
\begin{aligned}
P_{E_{f}}(\lambda) & =-\lambda^{5}+\left(-d_{1}-d_{4}-d_{3}-k_{2}-d_{2}\right) \lambda^{4} \\
& +\left(-d_{1}\left(d_{4}+d_{3}+k_{2}+d_{2}\right)-d_{4} d_{3}-d_{4} k_{2}-d_{4} d_{2}-d_{3} k_{2}-d_{3} d_{2}\right) \lambda^{3} \\
& +\left(-d_{1}\left(d_{4} d_{3}+d_{4} k_{2}+d_{4} d_{2}+d_{3} k_{2}+d_{3} d_{2}\right)-d_{4} d_{3} k_{2}-d_{4} d_{3} d_{2}+a k_{2} k_{1}\right) \lambda^{2} \\
& -d_{1}\left(d_{4} d_{3} k_{2}+d_{4} d_{3} d_{2}-a k_{2} k_{1}\right) \lambda .
\end{aligned}
$$

Therefore,

$$
\begin{aligned}
P_{E_{f}}(\lambda) & =\left(\lambda+d_{1}\right)(\lambda+b)\left(\lambda^{3}+\left(d_{4}+d_{3}+k_{2}+d_{2}\right)\right. \\
\lambda^{2} & \left.+\left(d_{4} d_{3}+d_{4} k_{2}+d_{4} d_{2}+d_{3} k_{2}+d_{3} d_{2}\right) \lambda+d_{4} d_{3} k_{2}+d_{4} d_{3} d_{2}-a k_{2} k_{1}\right),
\end{aligned}
$$

and the two obvious first eigenvalues of the matrix $J_{E_{f}}$ are $-b$ and $-d_{1}$, which are non-positive. Denoting

$$
\begin{aligned}
& a_{1}=d_{4}+d_{3}+k_{2}+d_{2} \\
& a_{2}=d_{4} d_{3}+d_{4} k_{2}+d_{4} d_{2}+d_{3} k_{2}+d_{3} d_{2} \\
& a_{3}=d_{4} d_{3} k_{2}+d_{4} d_{3} d_{2}-a k_{2} k_{1}
\end{aligned}
$$


it is easy to check that we have always $a_{1}>0, a_{2}>0$ and $a_{1} a_{2}-a_{3}>0$. In addition, it is easy to see that $a_{3}>0$ when $R_{0}<1$. From the Routh-Hurwitz criterion, it follows that $E_{f}$ is locally asymptotically stable.

\section{Appendix B. Global Stability Result Proof}

The global stability proof of the endemic point $E_{f}$ (Proposition 3 ) is given as follows. Consider the following Lyapunov function:

$$
\mathcal{L}(x, y, s, v, z)=s+\frac{d_{2}+k_{2}}{k_{2}} y+\frac{d_{3}\left(d_{2}+k_{2}\right)}{a k_{2}} v+\frac{p}{c} \frac{d_{2}+k_{2}}{k_{2}} z .
$$

The time derivative is given by:

$$
\begin{aligned}
\dot{\mathcal{L}}(x, y, s, v, z) & =\dot{s}+\frac{d_{2}+k_{2}}{k_{2}} \dot{y}+\frac{d_{3}\left(d_{2}+k_{2}\right)}{a k_{2}} \dot{v}+\frac{p}{c} \frac{d_{2}+k_{2}}{k_{2}} \dot{z} . \\
\dot{\mathcal{L}}(x, y, s, v, z) & =\frac{k_{1} x v}{x+v}-\left(d_{2}+k_{2}\right) s+\frac{d_{2}+k_{2}}{k_{2}}\left(k_{2} s-d_{3} y\right)-\frac{d_{2}+k_{2}}{k_{2}} p y z \\
& +\frac{d_{3}\left(d_{2}+k_{2}\right)}{a k_{2}}\left(a y-d_{4} v\right)+\frac{p}{c} \frac{d_{2}+k_{2}}{k_{2}}(c y z-b z) \\
\dot{\mathcal{L}}(x, y, s, v, z) & =\frac{k_{1} x v}{x+v}-\frac{d_{3} d_{4}\left(d_{2}+k_{2}\right)}{a k_{2}} v-\frac{b p}{c} \frac{d_{2}+k_{2}}{k_{2}} z \\
\dot{\mathcal{L}}(x, y, s, v, z) & \leq k_{1} v-\frac{d_{3} d_{4}\left(d_{2}+k_{2}\right)}{a k_{2}} v \\
& \leq \frac{d_{3} d_{4}\left(d_{2}+k_{2}\right)}{a k_{2}}\left(R_{0}-1\right) v .
\end{aligned}
$$

If $R_{0}<1$, then $\dot{\mathcal{L}} \leq 0$. Moreover, $\dot{\mathcal{L}} \leq 0$ when $v=0$. The largest compact invariant is

$$
E=\left\{(x, y, s, v, z) \in \mathbb{R}_{*}^{+} \mid v=0\right\},
$$

according to LaSalle's invariance principle, $\lim _{+\infty} v(t)=0$, the limit system of equations is

$$
\left\{\begin{array}{l}
\dot{x}=\lambda-d_{1} x \\
\dot{y}=-d_{2} s-k_{2} s \\
\dot{s}=k_{2} s-d_{3} y-p y z \\
\dot{z}=b y z-c z .
\end{array}\right.
$$

We define another Lyapunov function and for implicity, using the same notation,

$$
\mathcal{L}(x, s, y, z)=\frac{1}{x_{0}}\left(x-x_{0}-x_{0} \ln \frac{x}{x_{0}}\right)+s+\frac{d_{2}+k_{2}}{k_{2}} y+\frac{p}{c} \frac{d_{2}+k_{2}}{k_{2}} z .
$$

Since $x_{0}=\frac{\lambda}{d_{1}}$, then

$$
\dot{\mathcal{L}}(x, s, y, z)=d_{1}\left(2-\frac{x}{x_{0}}-\frac{x_{0}}{x}\right)-\frac{d_{3}\left(d_{2}+k_{2}\right)}{k_{2}} s-\frac{p b}{c} z
$$

since the arithmetic mean is greater than or equal to the geometric mean, it follows that

$$
2-\frac{x}{x_{0}}-\frac{x_{0}}{x} \leq 0
$$

therefore, $\dot{\mathcal{L}} \leq 0$ and the equality holds if $x=x_{0}$ and $s=y=z=0$, which completes the proof. 
The global stability proof of the endemic point $E_{1}$ (Proposition 4 ) is given as follows. We employ the following Lyapunov function:

$$
\begin{aligned}
\mathcal{L}(x, y, s, v, z) & =x-x_{1}-\int_{x_{1}}^{x} \frac{\left(d_{2}+k_{2}\right) s_{1}}{\frac{k_{1} u v_{1}}{u+v_{1}}} d u+s-s_{1}-s_{1} \ln \frac{s}{s_{1}} \\
& +\frac{d_{2}+k_{2}}{k_{2}}\left(y-y_{1}-y_{1} \ln \frac{y}{y_{1}}\right)+\frac{d_{3}\left(d_{2}+k_{2}\right)}{a k_{2}}\left(v-v_{1}-v_{1} \ln \frac{v}{v_{1}}\right) \\
& +\frac{p}{c} \frac{d_{2}+k_{2}}{k_{2}} z .
\end{aligned}
$$

We have

$$
\begin{aligned}
\dot{\mathcal{L}}(x, y, s, v, z) & =\dot{x}-\left(d_{2}+k_{2}\right) s_{1} \frac{x+v_{1}}{k_{1} x v_{1}} \dot{x}+\dot{s}-\frac{s_{1}}{s} \dot{s}+\frac{d_{2}+k_{2}}{k_{2}}\left(\dot{y}-\frac{y_{1}}{y} \dot{y}\right) \\
& +\frac{d_{3}\left(d_{2}+k_{2}\right)}{a k_{2}}\left(\dot{v}-\frac{v_{1}}{v} \dot{v}\right)+\frac{p}{c} \frac{d_{2}+k_{2}}{k_{2}} \dot{z} .
\end{aligned}
$$

On the other hand, we have

$$
\left\{\begin{array}{l}
\lambda=d_{1} x_{1}+\left(d_{2}+k_{2}\right) s_{1} \\
\frac{k_{1} x_{1} v_{1}}{x_{1}+v_{1}}=\left(d_{2}+k_{2}\right) s_{1}, \\
\frac{s_{1}}{v_{1}}=\frac{d_{3} d_{4}}{a k_{2}}, \frac{y_{1}}{v_{1}}=\frac{d_{4}}{a}, \frac{s_{1}}{y_{1}}=\frac{d_{3}}{k_{2}} .
\end{array}\right.
$$

Hence,

$$
\begin{aligned}
\dot{\mathcal{L}}(x, s, y, v, z) & =\left(\lambda-d_{1} x-\frac{k_{1} x v}{x+v}\right)\left(1-\left(d_{2}+k_{2}\right) s_{1} \frac{x+v_{1}}{k_{1} x v_{1}}\right) \\
& +\frac{k_{1} x v}{x+v}-\left(d_{2}+k_{2}\right) s-\frac{s_{1}}{s}\left(\frac{k_{1} x v}{x+v}-\left(d_{2}+k_{2}\right) s\right) \\
& +\frac{d_{2}+k_{2}}{k_{2}}\left(k_{2} s-d_{3} y-p y z\right)-\frac{d_{2}+k_{2}}{k_{2}} \frac{y_{1}}{y}\left(k_{2} s-d_{3} y-p y z\right) \\
& +\frac{d_{3}\left(d_{2}+k_{2}\right)}{a k_{2}}\left(a y-d_{4} v\right)-\frac{d_{3}\left(d_{2}+k_{2}\right)}{a k_{2}} \frac{v_{1}}{v}\left(a y-d_{4} v\right) \\
& +\frac{p}{c} \frac{d_{2}+k_{2}}{k_{2}}(c y z-b z),
\end{aligned}
$$

and

$$
\begin{aligned}
\dot{\mathcal{L}}(x, s, y, v, z)= & \lambda-d_{1} x-\left(d_{2}+k_{2}\right) s_{1} \frac{x+v_{1}}{k_{1} x v_{1}}\left(\lambda-d_{1} x-\frac{k_{1} x v}{x+v}\right) \\
& -\frac{s_{1}}{s}\left(\frac{k_{1} x v}{x+v}\right)+\left(d_{2}+k_{2}\right) s_{1}+\frac{d_{2}+k_{2}}{k_{2}} p y_{1} z \\
& +\frac{\left(d_{2}+k_{2}\right) d_{3}}{k_{2}} y_{1}-\left(d_{2}+k_{2}\right) s \frac{y_{1}}{y}-\frac{d_{3} d_{4}\left(d_{2}+k_{2}\right)}{a k_{2}} v \\
& +\frac{d_{3} d_{4}\left(d_{2}+k_{2}\right)}{a k_{2}} v_{1}-\frac{d_{3}\left(d_{2}+k_{2}\right)}{k_{2}} \frac{v_{1} y}{v}-\frac{d_{2}+k_{2}}{k_{2}} \frac{b p}{c} z .
\end{aligned}
$$


Since

$$
\left\{\begin{array}{l}
\lambda-d_{1} x=d_{1} x_{1}+\left(d_{2}+k_{2}\right) s_{1}-d_{1} x, \\
\lambda-d_{1} x-\left(d_{2}+k_{2}\right) s_{1} \frac{x+v_{1}}{k_{1} x v_{1}}\left(\lambda-d_{1} x-\frac{k_{1} x v}{x+v}\right)=d_{1} x_{1}\left(1-\frac{x}{x_{1}}-\frac{x_{1}}{x} \frac{x+v_{1}}{x_{1}+v_{1}}+\frac{x+v_{1}}{x_{1}+v_{1}}\right) \\
\quad+\left(d_{2}+k_{2}\right) s_{1}\left(1-\frac{x_{1}}{x} \frac{x+v_{1}}{x_{1}+v_{1}}+\frac{v}{v_{1}} \frac{x+v_{1}}{x+v}\right), \\
-\frac{s_{1}}{s}\left(\frac{k_{1} x v}{x+v}\right)+\left(d_{2}+k_{2}\right) s_{1}=-\frac{s_{1}}{s} \frac{x v}{x_{1} v_{1}} \frac{x_{1}+v_{1}}{x+v}\left(d_{2}+k_{2}\right) s_{1}+\left(d_{2}+k_{2}\right) s_{1},
\end{array}\right.
$$

we have

$$
\begin{aligned}
\dot{\mathcal{L}} & =d_{1} x_{1}\left(1-\frac{x}{x_{1}}-\frac{x_{1}}{x} \frac{x+v_{1}}{x_{1}+v_{1}}+\frac{x+v_{1}}{x_{1}+v_{1}}\right) \\
& +\left(d_{2}+k_{2}\right) s_{1}\left(1-\frac{x_{1}}{x} \frac{x+v_{1}}{x_{1}+v_{1}}+\frac{v}{v_{1}} \frac{x+v_{1}}{x+v}\right) \\
& +\left(d_{2}+k_{2}\right) s_{1}\left(1-\frac{s_{1}}{s} \frac{x v}{x_{1} v_{1}} \frac{x_{1}+v_{1}}{x+v}\right) \\
& +\left(d_{2}+k_{2}\right) s_{1}\left(1-\frac{s y_{1}}{s_{1} y}-\frac{v}{v_{1}}\right) \\
& +\left(d_{2}+k_{2}\right) s_{1}\left(1-\frac{v_{1} y}{y_{1} v}\right)+p z \frac{d_{2}+k_{2}}{k_{2}}\left(y_{1}-\frac{b}{c}\right) .
\end{aligned}
$$

Therefore,

$$
\begin{aligned}
\dot{\mathcal{L}} & =-\frac{d_{1} v_{1}}{x\left(x_{1}+v_{1}\right)}\left(x-x_{1}\right)^{2} \\
& +\left(d_{2}+k_{2}\right) s_{1}\left(-1-\frac{v}{v_{1}}+\frac{v}{v_{1}} \frac{x+v_{1}}{x+v}+\frac{x+v}{x+v_{1}}\right) \\
& +\left(d_{2}+k_{2}\right) s_{1}\left(5-\frac{x_{1}}{x} \frac{x+v_{1}}{x_{1}+v_{1}}-\frac{s_{1}}{s} \frac{x v}{x_{1} v_{1}} \frac{x_{1}+v_{1}}{x+v}-\frac{s y_{1}}{s_{1} y}-\frac{y v_{1}}{y_{1} v}-\frac{x+v}{x+v_{1}}\right) \\
& +p z \frac{d_{2}+k_{2}}{k_{2}}\left(y_{1}-\frac{b}{c}\right),
\end{aligned}
$$

which implies that

$$
\begin{aligned}
\dot{\mathcal{L}} & =-\frac{d_{1} v_{1}}{x\left(x_{1}+v_{1}\right)}\left(x-x_{1}\right)^{2} \\
& -\left(d_{2}+k_{2}\right) s_{1}\left(\frac{x\left(v-v_{1}\right)^{2}}{v_{1}\left(x+v_{1}\right)(x+v)}\right) \\
& +\left(d_{2}+k_{2}\right) s_{1}\left(5-\frac{x_{1}}{x} \frac{x+v_{1}}{x_{1}+v_{1}}-\frac{s_{1}}{s} \frac{x v}{x_{1} v_{1}} \frac{x_{1}+v_{1}}{x+v}-\frac{s y_{1}}{s_{1} y}-\frac{y v_{1}}{y_{1} v}-\frac{x+v}{x+v_{1}}\right) \\
& +p z \frac{d_{2}+k_{2}}{k_{2}}\left(y_{1}-\frac{b}{c}\right),
\end{aligned}
$$

since the arithmetic mean is greater than or equal to the geometric mean, it follows that

$$
5-\frac{x_{1}}{x} \frac{x+v_{1}}{x_{1}+v_{1}}-\frac{s_{1}}{s} \frac{x v}{x_{1} v_{1}} \frac{x_{1}+v_{1}}{x+v}-\frac{s y_{1}}{s_{1} y}-\frac{y v_{1}}{y_{1} v}-\frac{x+v}{x+v_{1}} \leq 0 .
$$

In addition, when $R_{C T L}<1$, we will have $y_{1}-\frac{b}{c} \leq 0$, which means that $\dot{L} \leq 0$, and the equality hods when $x=x_{1}, y=y_{1}, s=s_{1}$ and $v=v_{1}$. By the LaSalle invariance principle, the endemic point $E_{1}$ is globally stable when $R_{0}>1$. 
Finally, the global stability proof of the endemic point $E_{2}$ (Proposition 5) is given as follows. We employ the following Lyapunov function:

$$
\begin{aligned}
\mathcal{L}(x, s, y, v, z)= & -x_{2}-\int_{x_{2}}^{x} \frac{\left(d_{2}+k_{2}\right) s_{2}}{\frac{k_{1} u v_{2}}{u+v_{2}}} d u+s-s_{2}-s_{2} \ln \frac{s}{s_{2}} \\
& +\frac{d_{2}+k_{2}}{k_{2}}\left(y-y_{2}-y_{2} \ln \frac{y}{y_{2}}\right)+\frac{d_{3}\left(d_{2}+k_{2}\right)+\left(d_{2}+k_{2}\right) p z_{2}}{a k_{2}} \\
& \times\left(v-v_{2}-v_{2} \ln \frac{v}{v_{2}}\right)+\frac{p}{c} \frac{d_{2}+k_{2}}{k_{2}}\left(z-z_{2}-z_{2} \ln \frac{z}{z_{2}}\right) .
\end{aligned}
$$

We have

$$
\begin{aligned}
\dot{\mathcal{L}}(x, y, s, v, z)= & \dot{x}-\left(d_{2}+k_{2}\right) s_{2} \frac{x+v_{2}}{k_{1} x v_{2}} \dot{x}+\dot{s}-\frac{s_{2}}{s} \dot{s}+\frac{d_{2}+k_{2}}{k_{2}}\left(\dot{y}-\frac{y_{2}}{y} \dot{y}\right) \\
& +\frac{d_{3}\left(d_{2}+k_{2}\right)+\left(d_{2}+k_{2}\right) p z_{2}}{a k_{2}}\left(\dot{v}-\frac{v_{2}}{v} \dot{v}\right)+\frac{p}{c} \frac{d_{2}+k_{2}}{k_{2}}\left(\dot{z}-\frac{z_{2}}{z} \dot{z}\right) .
\end{aligned}
$$

Since,

$$
\left\{\begin{array}{l}
\lambda=d_{1} x_{2}+\left(d_{2}+k_{2}\right) s_{2} \\
k_{1} x_{2} v_{2}=\left(x_{2}+v_{2}\right)\left(d_{2}+k_{2}\right) s_{2} \\
\frac{s_{2}}{y_{2}}=\frac{d_{3}}{k_{2}}+\frac{p z_{2}}{k_{2}}, \frac{y_{2}}{v_{2}}=\frac{d_{4}}{a}, \frac{s_{2}}{v_{2}}=\frac{d_{3} d_{4}}{a k_{2}}+\frac{d_{4} p z_{2}}{a k_{2}}
\end{array}\right.
$$

we have

$$
\begin{aligned}
\dot{\mathcal{L}}(x, s, y, v, z)= & \lambda-d_{1} x-\frac{x_{2}}{x} \frac{x+v_{2}}{x_{2}+v_{2}}\left(\lambda-d_{1} x\right)+\left(\left(d_{2}+k_{2}\right) s_{2}\right) \frac{v}{v_{2}} \frac{x_{2}+v_{2}}{x+v}-\left(d_{2}+k_{2}\right) s \\
& -\frac{s_{2}}{s}\left(\frac{k_{1} x v}{x+v}-\left(d_{2}+k_{2}\right) s\right)+\frac{d_{2}+k_{2}}{k_{2}}\left(k_{2} s-d_{3} y-p y z\right) \\
& -\frac{d_{2}+k_{2}}{k_{2}} \frac{y_{2}}{y}\left(k_{2} s-d_{3} y-p y z\right)+\frac{d_{3}\left(d_{2}+k_{2}\right)+\left(d_{2}+k_{2}\right) p z_{2}}{a k_{2}} \\
& \times\left(a y-d_{4} v\right)-\frac{d_{3}\left(d_{2}+k_{2}\right)+\left(d_{2}+k_{2}\right) p z_{2}}{a k_{2}} \frac{v_{2}}{v}\left(a y-d_{4} v\right) \\
& +\frac{p}{c} \frac{d_{2}+k_{2}}{k_{2}}(c y z-b z)-\frac{p z_{2}}{c z} \frac{d_{2}+k_{2}}{k_{2}}(c y z-b z) .
\end{aligned}
$$

On the other hand, we have

$$
\left\{\begin{array}{l}
\left.\lambda-d_{1} x=d_{1} x_{2}+d_{2}+k_{2}\right) s_{2}-d_{1} x \\
\lambda-d_{1} x-\left(d_{2}+k_{2}\right) s_{2} \frac{x+v_{2}}{k_{1} x v_{2}}\left(\lambda-d_{1} x-\frac{k_{1} x v}{x+v}\right)=d_{1} x_{2}\left(1-\frac{x}{x_{2}}-\frac{x_{2}}{x} \frac{x+v_{2}}{x_{2}+v_{2}}\right. \\
\left.\quad+\frac{x+v_{2}}{x_{2}+v 2}\right)+\left(d_{2}+k_{2}\right) s_{2}\left(1-\frac{x_{2}}{x} \frac{x+v_{2}}{x_{2}+v_{2}}+\frac{v}{v_{2}} \frac{x+v_{2}}{x+v}\right) \\
-\frac{s_{2}}{s}\left(\frac{k_{1} x v}{x+v}\right)+\left(d_{2}+k_{2}\right) s_{2}=\left(d_{2}+k_{2}\right) s_{2}\left(1-\frac{s_{2}}{s} \frac{x v}{x_{2} v_{2}} \frac{x_{2}+v_{2}}{x+v}\right),
\end{array}\right.
$$

and

$$
\left\{\begin{array}{l}
\frac{\left(d_{2}+k_{2}\right) d_{3}}{k_{2}} y_{2}-\left(d_{2}+k_{2}\right) s \frac{y_{2}}{y}-\frac{\left(d_{2}+k_{2}\right) d_{3} d_{4}}{a k_{2}} v \\
=\left(d_{2}+k_{2}\right) s_{2}\left(1-\frac{s}{s_{2}} \frac{y_{2}}{y}-\frac{v}{v_{2}}\right)+\frac{\left(d_{2}+k_{2}\right)}{k_{2}} p z_{2} y_{2} \frac{v}{v_{2}}-\frac{\left(d_{2}+k_{2}\right)}{k_{2}} p z_{2} y_{2} \\
\frac{d_{4} d_{3}\left(d_{2}+k_{2}\right)}{a k_{2}} v_{2}-\frac{d_{3}\left(d_{2}+k_{2}\right)}{k_{2}} \frac{v_{2}}{v} y=\left(d_{2}+k_{2}\right) s_{2}\left(1-\frac{y}{y_{2}} \frac{v_{2}}{v}\right) \\
\quad+\frac{\left(d_{2}+k_{2}\right)}{k_{2}} p z_{2} y \frac{v_{2}}{v}-\frac{\left(d_{2}+k_{2}\right)}{k_{2}} p z_{2} y_{2}
\end{array}\right.
$$




$$
\left\{\begin{array}{l}
\frac{\left(d_{2}+k_{2}\right) p z_{2}}{a k_{2}}\left(a y-d_{4} v\right)-\frac{\left(d_{2}+k_{2}\right) p z_{2}}{a k_{2}} \frac{v_{2}}{v}\left(a y-d_{4} v\right)=\frac{\left(d_{2}+k_{2}\right) p z_{2} y}{k_{2}} \\
\quad-\frac{\left(d_{2}+k_{2}\right) p z_{2} y_{2}}{k_{2}} \frac{v}{v_{2}}-\frac{\left(d_{2}+k_{2}\right) p z_{2} y}{k_{2}} \frac{v_{2}}{v}+\frac{\left(d_{2}+k_{2}\right) p z_{2} y_{2}}{k_{2}} \\
\frac{\left(d_{2}+k_{2}\right) p z_{2}}{a k_{2}}\left(a y-d_{4} v\right)-\frac{\left(d_{2}+k_{2}\right) p z_{2}}{a k_{2}} \frac{v_{2}}{v}\left(a y-d_{4} v\right)-\frac{z_{2}}{z} \frac{p}{c} \frac{d_{2}+k_{2}}{k_{2}}(x y z-b z) \\
+\frac{\left(d_{2}+k_{2}\right)}{k_{2}} p z_{2} y_{2} \frac{v}{v_{2}}-\frac{\left(d_{2}+k_{2}\right)}{k_{2}} p z_{2} y_{2}+\frac{\left(d_{2}+k_{2}\right)}{k_{2}} p z_{2} y \frac{v_{2}}{v}-\frac{\left(d_{2}+k_{2}\right)}{k_{2}} p z_{2} y_{2}=0 .
\end{array}\right.
$$

All of these imply

$$
\begin{aligned}
\dot{L} & =-\frac{d_{1} v_{2}}{x\left(x_{2}+v_{2}\right)}\left(x-x_{2}\right)^{2} \\
& -\left(d_{2}+k_{2}\right) s_{2}\left(\frac{x\left(v-v_{2}\right)^{2}}{v_{2}\left(x+v_{2}\right)(x+v)}\right) \\
& +\left(d_{2}+k_{2}\right) s_{2}\left(5-\frac{x_{2}}{x} \frac{x+v_{2}}{x_{2}+v_{2}}-\frac{s_{2}}{s} \frac{x v}{x_{2} v_{2}} \frac{x_{2}+v_{2}}{x+v}-\frac{s y_{2}}{s_{2} y}-\frac{y v_{2}}{y_{2} v}-\frac{x+v}{x+v_{2}}\right) .
\end{aligned}
$$

Again, since the arithmetic mean is greater than or equal to the geometric mean, it follows that

$$
5-\frac{x_{2}}{x} \frac{x+v_{2}}{x_{2}+v_{2}}-\frac{s_{2}}{s} \frac{x v}{x_{2} v_{2}} \frac{x_{2}+v_{2}}{x+v}-\frac{s y_{2}}{s_{2} y}-\frac{y v_{2}}{y_{2} v}-\frac{x+v}{x+v_{2}} \leq 0,
$$

which means that $\dot{L} \leq 0$, and the equality hods when $x=x_{2}, s=s_{2}, y=y_{2}, v=v_{2}$ and $z=z_{2}$. By the LaSalle invariance principle, the endemic point $E_{2}$ is globally stable.

\section{References}

1. $\quad$ Blanttner, W.; Gallo, R.C.; Temin, H.M. HIV causes aids. Science 1988, 241, 515-516.

2. Weiss, R.A. How does HIV cause AIDS? Science 1993, 260, 1273-1279.

3. Saez-Cirion, A.; Lacabaratz, C.; Lambotte, O.; Versmisse, P.; Urrutia, A.; Boufassa, F.; Barre-Sinoussi, F.; Delfraissy, J.F.; Sinet, M.; Pancino, G.; et al. HIV controllers exhibit potent CD8 T cell capacity to suppress HIV infection ex vivo and peculiar cytotoxic T-lymphocyte activation phenotype. Proc. Natl. Acad. Sci. USA 2007, 104, 6776-6781.

4. Betts, M.R.; Nason, M.C.; West, S.M.; De Rosa, S.C.; Migueles, S.A.; Abraham, J.; Lederman, M.M.; Benito, J.M.; Goepfert, P.A.; Connors, M.; et al. HIV nonprogressors preferentially maintain highly functional HIV-specific CD8 ${ }^{+}$T-cells. Blood 2006, 107, 4781-4789.

5. Nowak, M.A.; Bangham, C.R.M. Population dynamics of immune responses to persistent viruses. Science 1996, 272, 74-79.

6. Smith, H.L.; De Leenheer, P. Virus dynamics: A global analysis. SIAM J. Appl. Math. 2003, 63, 1313-1327.

7. Korobeinikov, A. Global properties of basic virus dynamics models. Bull. Math. Biol. 2004, 66, 879-883.

8. Daar, E.S.; Moudgil, T.; Meyer, R.D.; Ho, D.D. Transient highlevels of viremia in patients with primary human immunodeficiency virus type 1 infection. N. Engl. J. Med. 1991, 324, 961-964.

9. Kahn, J.O.; Walker, B.D. Acute human immunodeficiency virus type 1 infection. N. Engl. J. Med. 1998, 339, 33-39.

10. Kaufmann, G.R.; Cunningham, P.; Kelleher, A.D.; Zauders, J.; Carr A.; Vizzard, J.; Law, M.; Cooper, D.A. Patterns of viral dynamics during primary human immunodeficiency virus type 1 infection. J. Infect. Dis. 1998, 178, 1812-1815.

11. Schacker, T.; Collier, A.; Hughes, J.; Shea, T.; Corey, L. Clinical and epidemiologic features of primary HIV infection. Ann. Int. Med. 1996, 125, 257-264.

12. Wang, X.; Elaiw, A.; Song, X. Global properties of a delayed HIV infection model with CTL immune response. Appl. Math. Comput. 2012, 218, 9405-9414.

13. Wang, X.; Tao, Y.; Song, X. Global stability of a virus dynamics model with Beddington-DeAngelis incidence rate and CTL immune response. Nonlinear Dyn. 2011, 66, 825-830. 
14. Sun, Q.; Min, L.; Kuang, Y. Global stability of infection-free state and endemic infection state of a modified human immunodeficiency virus infection model. IET Syst. Biol. 2015, 9, 95-103.

15. Sun, Q.; Min, L. Dynamics Analysis and Simulation of a Modified HIV Infection Model with a Saturated Infection Rate. Comput. Math. Methods Med. 2014, 2014, 145162.

16. Wang, Y.; Zhou, Y.; Wu, J.; Heffernan, J. Oscillatory viral dynamics in a delayed HIV pathogenesis model. Math. Biosci. 2009, 219, 104-112.

17. Zhu, H.; Luo, Y.; Chen, M. Stability and Hopf bifurcation of a HIV infection model with CTL-response delay. Comput. Math. Appl. 2011, 62, 3091-3102.

18. De Boer, R.J.; Perelson, A.S. Target cell limited and immune control models of HIV infection: A comparison. J. Theor. Biol. 1998, 190, 201-214.

19. Conway, J.M.; Perelson, A.S. Post-treatment control of HIV infection. Proc. Natl. Acad. Sci. USA 2015, 112, 5467-5472.

(C) 2017 by the authors. Licensee MDPI, Basel, Switzerland. This article is an open access article distributed under the terms and conditions of the Creative Commons Attribution (CC BY) license (http:/ / creativecommons.org/licenses/by/4.0/). 